\title{
Image-Guided Percutaneous Ablation of Hepatic Malignancies
}

\author{
Gretchen Foltz, MD ${ }^{1}$ \\ ${ }^{1}$ Division of Interventional Radiology, Mallinckrodt Institute of \\ Radiology, St. Louis, Missouri
}

Semin Intervent Radiol 2014;31:180-186

\begin{abstract}
Address for correspondence Gretchen Foltz, MD, Division of Interventional Radiology, Mallinckrodt Institute of Radiology, 510 South Kingshighway Boulevard, St. Louis, MO 63110 (e-mail: foltzg@mir.wustl.edu).
\end{abstract}

\begin{abstract}
Keywords

- percutaneous ablation

- hepatocellular carcinoma

- hepatic metastases

- interventional radiology

The liver is a common site of primary and secondary malignancies, often resulting in significant morbidity and mortality. Evaluating these patients in a multidisciplinary setting allows for optimal utilization of all oncologic therapies including surgery, radiation, systemic chemotherapy, transarterial therapies, and ablation. While surgical intervention often provides the best outcomes when treating most hepatic tumors, many patients are not surgical candidates due to extensive tumor burden, underlying liver disease, or other comorbid conditions. The evolution of imaging and ablation devices has allowed for the increased utilization of percutaneous ablation as definitive and palliative treatment of primary and metastatic hepatic malignancies. Ablation induces tumor necrosis by injection of chemicals (chemical ablation) or temperature modification (thermal ablation). The goal of this review is to provide an overview of different ablation techniques commonly used for hepatic malignancies, discuss the oncologic outcomes of these interventions, and outline the current indications, contraindications, and reported complications of these therapies.
\end{abstract}

Objectives: Upon completion of this article, the reader will be able to identify appropriate patient selection for percutaneous ablation, methods commonly used for thermal and chemical ablation, and expected oncologic outcome of these therapies.

Accreditation: This activity has been planned and implemented in accordance with the Essential Areas and Policies of the Accreditation Council for Continuing Medical Education (ACCME) through the joint providership of Tufts University School of Medicine (TUSM) and Thieme Medical Publishers, New York. TUSM is accredited by the ACCME to provide continuing medical education for physicians.

Credit: Tufts University School of Medicine designates this journal-based CME activity for a maximum of 1 AMA PRA Category 1 Credit $^{\mathrm{TM}}$. Physicians should claim only the credit commensurate with the extent of their participation in the activity.

The concept of tumor ablation has existed in medicine for over a century. ${ }^{1}$ However, the percutaneous application of ablation therapies did not widely emerge until the 1980s. Early percutaneous approaches to primary hepatic malignancy focused on the use of ethanol administered under ultrasound guidance. ${ }^{2}$ Since then, numerous thermal ablation modalities have been developed and studied, resulting in a growing body of clinical research. Given that primary and secondary hepatic malignancies are common and a significant source of mortality, it is not surprising that minimally invasive treatment options have gained popularity.

Hepatocellular carcinoma (HCC) is the sixth most common cancer worldwide and the third most common cause of cancer mortality. ${ }^{3}$ HCC is also the leading cause of death among patients with cirrhosis. ${ }^{4}$ Metastatic tumors of the liver are even more common, especially in the setting of colorectal carcinoma. Twenty percent of patients with colorectal cancer have evidence of liver metastases at diagnosis, and 50\% will develop hepatic metastatic disease over the course of their illness. $^{5}$

For various reasons, the majority of patients with primary or secondary hepatic malignancies are not candidates for
Issue Theme Tumor Ablation; Guest Editor, Charles T. Burke, MD, FSIR
Copyright @ 2014 by Thieme Medical Publishers, Inc., 333 Seventh Avenue, New York, NY 10001, USA. Tel: +1(212) 584-4662.
DOI http://dx.doi.org/ $10.1055 / \mathrm{s}-0034-1373792$. ISSN 0739-9529. 
surgery. Percutaneous ablation has been shown to be a viable option for the treatment in some of these patients. This review focuses on image-guided tumor ablation for local control of early-stage HCC and small liver metastases in surgically unresectable patients.

\section{Patient Selection for Percutaneous Ablation}

Given the wide range of treatment options available for patients with hepatic malignancies, patients are often discussed and triaged in multidisciplinary settings with the goal of obtaining the best oncologic outcome for each individual. Patient prognosis is guided by staging. There are several staging systems available for HCC, with no single system being universally superior to the others. The Barcelona Clinic Liver Cancer (BCLC) and the United Network for Organ Sharing (UNOS) classification systems are commonly used and referenced in treatment guidelines. BCLC is a staging system for HCC that incorporates tumor burden, liver functional reserve, and general performance status. The main advantage of the BCLC staging system is that it links staging with treatment modalities, and with an estimation of life expectancy based on published response rates. While there are many factors that influence therapeutic decisions, this staging system has come to be widely accepted in western clinical practice and is referenced in many guidelines involving the treatment of $\mathrm{HCC}^{6-8}$

The UNOS staging system is used in many transplant centers. Liver transplant remains the most successful treatment for HCC, with reported 5-year survival rates in the range of 71 to $75 \% .^{9,10}$ Unfortunately, the number of patients awaiting transplant is significantly greater than the number of available organs. Although outcomes are not as favorable as those for transplant, surgical resection for HCC has 5-year survival rates in the range of 41 to $51 \% .^{11}$ However, less than $5 \%$ of patients with HCC meet criteria for resection.

According to the Barcelona Clinic Liver Cancer (BCLC) classification, ablation is the therapy of choice for patients with very early and early HCC who are not candidates for either liver resection or transplant. The best results have been in patients with: single tumors smaller than $5 \mathrm{~cm}$ or up to three tumors each smaller than $3 \mathrm{~cm}$, no evidence of vascular invasion or extra hepatic disease, and liver cirrhosis in ChildPugh class A or B. ${ }^{7,8}$

The prognosis for secondary hepatic tumors is largely dependent on the extent of the metastatic disease. In patients with colorectal metastases, nearly $25 \%$ will have metastatic disease isolated to the liver. Complete surgical resection is currently considered the curative treatment in this setting; however, only approximately $25 \%$ of patients are candidates. Ablation is generally indicated for nonsurgical patients with colorectal metastases isolated to the liver. Select patients with limited extrahepatic disease should also be considered for ablation treatment, provided that the extrahepatic disease is thought to be curable. Except for select patients (e.g., ablation performed for pain control rather than survival benefit), in the setting of multiple lesions ablation should only be considered if treatment of all metastatic lesions can be at- tempted. Most centers preferentially treat patients with five or fewer lesions, and the best results are in patients with tumors that are $3 \mathrm{~cm}$ or smaller. ${ }^{5,12}$ Patients with hepatic metastases from other primary malignancies have selection criteria similar to patients with colorectal metastatic disease.

Absolute contraindications to ablation therapy include: intravascular invasion, tumor location $<1 \mathrm{~cm}$ from the main biliary duct, intrahepatic biliary tree dilation, exophytic location of the tumor, or uncorrectable coagulopathy. Relative contraindications include: extrahepatic metastases, bilioenteric anastomosis, superficial lesions, lesions adjacent to any part of the gastrointestinal tract or gallbladder, ascites, platelet count $<50,000 / \mathrm{mm}^{3}$, Child-Pugh class C cirrhosis/ hepatic failure, or pacemaker/defibrillator.

\section{Percutaneous Ablation Modalities}

The most important goal of minimally invasive tumor ablation is to eradicate all viable malignant cells within a designated target volume. It has previously been demonstrated that viable malignant cells are present beyond visible tumor boundaries. Therefore, tumor ablation therapies are intended to include at least a 0.5 to $1.0 \mathrm{~cm}$ ablation margin of seemingly normal liver tissue in attempts to account for microsatellite lesions. ${ }^{13}$ While tumor eradication is of primary importance, sparing normal surrounding tissues is one potential advantage of ablative therapy over surgical resection; this allows for the possibility of repeat treatment if and when additional tumors arise.

\section{Chemical Ablation}

Percutaneous ablation was initially reported using chemical ablation techniques. Percutaneous ethanol injection (PEI) is principally used for the treatment of HCC in patients with cirrhosis. $^{14}$ The injection of ethanol into a tumor causes cellular dehydration, protein denaturation, and ultimately cell death. In addition, alcohol entering the local circulation leads to necrosis of the vascular endothelium and subsequent platelet aggregation, leading to vascular thrombosis and ultimately ischemic tissue necrosis. ${ }^{15}$ Under ultrasound guidance, a small gauge needle is used to inject 2 to $10 \mathrm{~mL}$ of $99.5 \%$ ethanol into the lesion. The number of treatments is dependent on the size of the lesion, although four to eight sessions are usually required. ${ }^{16,17}$ A capsule or pseudocapsule around HCCs allows increased concentration of the ethanol within the target lesion. PEI is used for lesions less than $3 \mathrm{~cm}$ due to limitations of uneven ethanol spread in larger lesions. ${ }^{13,16}$

The dense fibrous nature of metastatic lesions compared with the softer tissue of HCC results in poor diffusion of the chemical within these target lesions. In addition, metastatic lesions lack a capsule allowing diffusion of the chemical into the surrounding liver thereby lowering its efficacy. ${ }^{13,18} \mathrm{PEI}$ is therefore not effective and should not be used in the treatment of metastatic lesions.

Historically, PEI has been a widely used and studied ablation technique. Several studies demonstrate that longterm survival is greater in patients treated with PEI compared with patients receiving best supportive care. ${ }^{14,19}$ For small 
HCC $(<2 \mathrm{~cm})$, PEI has been shown to have 5-year survival rates near $50 \%{ }^{14,20}$

There have been several randomized controlled trials showing the superiority of radiofrequency ablation (RFA) to PEI. The rates of complete tumor ablation have been shown to be between 96 and 100\% for the RFA groups compared with 88 and $100 \%$ for the PEI groups, with significantly fewer treatment sessions required for the RFA groups. The local recurrence rates are reportedly between 2 and $17 \%$ for the RFA groups compared with 11 and $45 \%$ for the PEI groups. The 3-year survival was 74 to $80 \%$ and 50 to $63 \%$ for the RFA and PEI groups, respectively. ${ }^{17}$ Based on these studies, chemical ablation has largely been replaced with thermal ablation techniques in regions where it is available and locations amenable to thermal techniques.

\section{Thermal Ablation}

The goal of thermal ablation is to destroy tumor tissue by increasing or decreasing the temperature to induce irreversible cellular injury. Three phenomena define the results of hyperthermal ablation: heat production, heat conduction, and heat convection. ${ }^{13}$ Heat production is proportional to the energy delivered and the energy interaction with the target tissue; conduction is how energy spreads to the surrounding tissue; and convection is the decay of heat by local blood flow, also referred to as the heat sink effect. The goal of hyperthermal ablation is to destroy tumors by heating cells to more than 50 to $60^{\circ} \mathrm{C}$, causing irreversible cell death. In contrast, thermal ablation with cryoablation achieves cell death at -20 to $-40^{\circ} \mathrm{C}^{21}$

\section{Radiofrequency Ablation}

RFA is currently the most commonly used liver-directed ablative therapy. ${ }^{13}$ Radiofrequency energy is delivered as an alternating current at a frequency of $400 \mathrm{MHz}$, resulting in ionic frictional agitation and heat generation known as the Joule effect. The increase in the tissue temperature ultimately causes protein coagulation and tissue death. Tissues nearest to an electrode are heated most effectively, while more peripheral areas receive heat by thermal conduction. ${ }^{13}$ To increase the volume of ablated tissues, various applicators have been developed. Electrodes can be monopolar or bipolar and come in a variety of designs including: multitined expandable, internally cooled, and perfused. These electrode innovations result in ablation zones large enough to enable RFA treatment of tumors in the range of 2 to $5 \mathrm{~cm} .{ }^{22}$ Due to the physical limitations in energy deposition, tumor size has a significant impact on the effectiveness of RFA in local tumor control. Tumors are typically defined as small $(3.0 \mathrm{~cm}$ or smaller), intermediate $(3.1-5.0 \mathrm{~cm})$, and large $(5.1 \mathrm{~cm}$ or larger). Local control rates of $90 \%$ or higher have been shown for small tumors, compared with 71 and $6 \%$ for medium and large tumors, respectively. ${ }^{23}$ The location of the tumor in relation to vascular structures has also been shown to affect ablation success. Large vascular structures adjacent to the neoplastic tissue can have a cooling effect, often referred to as the heat sink effect; vessels greater than $3 \mathrm{~mm}$ in diameter resist thrombosis when exposed to the thermal energy and protect tissues within a few millimeters of the vessel lumen. ${ }^{24}$
It is difficult to use a standard scale to evaluate the outcomes of ablation therapies due to the heterogeneity of the patient population being treated and the overall lack of randomized data. Most studies for thermal ablation techniques are performed in patients who are not surgical candidates, and many have been treated with chemotherapy before ablation, especially in the setting of metastatic disease. Evaluation of treatment outcomes in patients with HCC is also complicated by the fact that in western countries, most HCCs arise in a background of cirrhosis, which has its own associated mortality risk.

There have been several long-term survival outcome studies of RFA in the treatment of HCC. These studies have helped define factors that can predict patient prognosis. The severity of the underlying cirrhosis and the number of tumors appear to be the most important prognostic factors. In a prospective, intention-to-treat analysis of RFA use in HCC reported by Lencioni et al, survival depended on the severity of the underlying liver disease and on tumor multiplicity. Patients with Child class A cirrhosis had 3- and 5-year survival rates of 76 and $51 \%$, respectively, significantly higher than the survival rates obtained for patients with Child class B cirrhosis (46\% at 3 years and $31 \%$ at 5 years). Patients with a solitary HCC had 3- and 5-year survival rates of 75 and 50\%, respectively, while patients with multiple tumors had 3- and 5-year survival rates of 51 and $34 \%$, respectively. ${ }^{11}$ A study by Choi et al demonstrated similar results with 3 - and 5-year survival rates of 78 and $64 \%$, respectively, in patients with Child class A cirrhosis compared with 3- and 5-year survival rates of 49 and $38 \%$, respectively, in patients with Child class B cirrhosis. This last study included patients with one lesion less than $5 \mathrm{~cm}$ or up to three lesions measuring $3 \mathrm{~cm}$ or less. ${ }^{25}$

There have been several retrospective studies where RFA was used in the treatment of colorectal liver metastases for patients who are nonsurgical candidates. For patients with five or fewer lesions, each $5 \mathrm{~cm}$ or less, the median survival varied between 24 and 52 months, with a 5-year overall survival of 18 to $44 \% .{ }^{26}$ Comparing RFA to surgical resection based on the available published data is difficult as there are no prospective randomized studies and patients treated with ablation are often considered to not be surgical candidates. Thus, it is not unexpected that the overall survival rate of RFA treated metastatic colorectal cancer patients (nonsurgical candidates) is lower when compared with a surgical resection group with a 5-year survival range between 18 and $40 \%$ in the RFA group and 23 and $58 \%$ in the surgical resection group. ${ }^{27,28}$

Not surprisingly, the factors reported to affect the success of RFA in colorectal metastases are similar to thermal ablation of HCC. Tumor size and location are strong predictors of success, with lesions smaller than $3 \mathrm{~cm}$ demonstrating less local recurrence compared with larger lesions. ${ }^{29} \mathrm{~A}$ higher local recurrence rate of $48 \%$ (as compared with $7 \%$ ) has been reported for tumors close to blood vessels. ${ }^{30}$

Hepatic metastases develop in approximately half of women with metastatic breast cancer and are associated with metastases at other sites, indicating advanced disease and poor prognosis. In patients receiving chemotherapy and supportive care in this setting, median overall survival ranges 
from 18 to 24 months. ${ }^{31}$ Patients with metastatic breast cancer have metastases confined to the liver in approximately $5 \%$ of cases, much lower than with colorectal metastatic disease. In patients with isolated liver metastatic lesions, median survival of 20 to 67 months and a 5-year survival rate of 21 to $61 \%$ have been reported following hepatic resection. In similar patient populations, several case series have demonstrated a median survival of 30 to 60 months after RFA for the treatment of liver metastases. ${ }^{32,33}$

Several case series of thermal ablation used to treat metastases from a variety of different primary tumor entities have been reported, including gastric cancer, ovarian cancer, and metastases of unknown primary. Although these series are small, there is a suggestion of potential survival benefit from percutaneous ablation treatment in patients who are otherwise not considered for surgery. ${ }^{26}$

\section{Microwave Ablation}

Although the cumulative reported experience with microwave ablation (MWA) is small, its use has been dramatically increasing in many practices. MWA results in hyperthermal cytotoxicity from the rapid oscillation of water molecules trying to align themselves in the alternating electric field, resulting in frictional heating. One or more antennae are placed into the lesion and are connected to a generator using a coaxial cable. Current microwave systems are available in two frequencies: $915 \mathrm{MHz}$ and $2.54 \mathrm{GHz}$. In contrast to RFA, there is no current conduction, so grounding pads are not needed. In addition, charring and vaporization in the vicinity of the needle are not obstacles to the delivery of energy. MWA also appears to be less susceptible to heat sink effects than RFA, and therefore may be more effective in treating tumors adjacent to larger vessels. ${ }^{34,35}$

Overall survival for patients with HCC treated with MWA has been evaluated in several studies. Liang et al have one of the largest series (288 patients) and includes patients with solitary lesions up to $8 \mathrm{~cm}$ as well as patients with up to 5 lesions (the largest of which was less than $6 \mathrm{~cm}$ ). The overall $1-, 3-$, and 5-year survival rates were 93,72 , and $51 \%$, respectively. The reported local recurrence rate was $8 \%{ }^{36}$ Dong et al demonstrated similar results in a series of 234 patients with 1-, 3-, and 5-year survival rates of 92.7, 72.8, and $56.7 \%$, respectively. There was no significant difference in survival between patients with lesions 3 to $5 \mathrm{~cm}$ and those with lesions 2 to $3 \mathrm{~cm}$ in size. However, the local recurrence rate was higher for those with tumors larger than $5 \mathrm{~cm}^{37}$

A few studies have directly compared MWA with RFA including a randomized study by Shibata et al. Inclusion criteria were solitary lesions less than $4 \mathrm{~cm}$ or two to three lesions less than or equal to $3 \mathrm{~cm}$. There was no significant difference in the rate of complete therapeutic effect (96\% for the RFA group and $89 \%$ for the MWA group). No survival rates were included with this analysis. ${ }^{38}$

\section{Cryoablation}

Cryoablation is one of the oldest thermal ablative techniques, first described in open surgical treatment of liver tumors in
1963. These were large probes that precluded percutaneous application. In the 1990s, advances in technology lead to the creation of smaller probes allowing for percutaneous placement. With cryoablation, tissue damage occurs through several mechanisms. Immediate cell death is caused by freezing and thawing cycles, creating a hyperosmotic environment and resulting in cell death by dehydration. Delayed tissue damage is caused by cellular anoxia from vascular stasis. ${ }^{21,39}$ Many of the commercially available cryoablation systems function by taking advantage of the Joule-Thompson effect. When gas is forced through a valve, it rapidly expands, leading to a decrease in kinetic energy and corresponding rapid decrease in temperature. Target temperatures are in the -20 to $-40^{\circ} \mathrm{C}$ range. The number of probes is determined by the size of the lesion; in general, the cryoprobes should be placed within $1 \mathrm{~cm}$ of the tumor edge and ideally spaced no further than 1.5 to $2 \mathrm{~cm}$ apart. ${ }^{40}$ At least two freeze-thaw cycles are generally performed. One advantage of cryoablation is that the ice ball can be imaged with computed tomography or ultrasound during treatment; the $-20^{\circ} \mathrm{C}$ isotherm marking the outer boundary of the zone of ablation is generally a few millimeters to a centimeter inside the outer boundary of the ice ball. ${ }^{41}$

Despite the availability of percutaneous cryoprobes, cryotherapy has not been as widely used in the treatment of hepatic tumors compared with RFA, and there are limited published data. Shimizu et al reported their experience with cryoablation in HCC in a small series, treating patients with one tumor no larger than $5 \mathrm{~cm}$ or multiple tumors less than $3 \mathrm{~cm}$. The 1- and 3-year overall survival rates were 93.8 and $79.3 \%$, respectively. ${ }^{42}$ Recently, a retrospective study comparing cryoablation to RFA was reported by Adam et al. A total of 64 patients with a combination of HCC and hepatic metastases were included. Overall 1-year survival rates were $73 \%$ in the cryoablation group and $60 \%$ in the RFA group. For patients with HCC, the 1 -year survival was 66 and $61 \%$ for cryoablation and RFA, respectively. In patients with metastases, 1-year survival rates were 84 and $58 \%$ for cryoablation and RFA, respectively. These results were not found to be significantly different. It was noted, however, that there was a slightly higher recurrence rate with cryoablation, particularly in the treatment of metastases. ${ }^{43}$

\section{Strategies to Improve Outcomes}

In attempts to improve survival in patients with medium and large size tumors, several studies have evaluated combination therapies. The most widely studied combinations are percutaneous ethanol instillation with RFA, and transcatheter arterial chemoembolization (TACE) with RFA. In general, the effectiveness of RFA is limited by tissue cooling secondary to tissue perfusion. Using ethanol before RFA causes tissue desiccation and destruction of small vessels, resulting in diminished tissue perfusion that leads to less tissue cooling and a larger zone of ablation. Similarly, TACE before RFA decreases blood flow through the tumor, facilitating a larger ablation zone. In addition, it is thought that the thermal therapy may potentiate the effects of chemotherapy. 
In a prospective randomized trial, Zhang et al showed percutaneous ethanol instillation combined with RFA resulted in no difference in 5-year survival for small tumors but a significant improvement in survival for intermediate size tumors (3.1-5.0 cm) compared with RFA alone. For those patients with intermediate-sized tumors, the 5-year survival rate was $56 \%$ in the combination group and $28 \%$ in the RFA alone group. ${ }^{44}$

Recently, a meta-analysis of RFA following TACE in the treatment of HCC that identified eight randomized controlled trials was published. The included studies had a combined total of 598 patients comparing RFA plus TACE with RFA alone. The tumor progression rate in patients treated with RFA alone was higher than with RFA plus TACE. There was no significant difference in major complications between the two groups. In addition, the meta-analysis data of subgroups revealed that the survival rate was significantly higher in patients with intermediate- and large-size HCC who underwent RFA plus TACE, compared with those who underwent RFA monotherapy. There was no significant difference on survival rates between RFA plus TACE and RFA for small HCC. ${ }^{45}$

Although the size of the lesion being treated has been shown to be a significant factor in survival outcome, the location of the lesion sometimes limits treatment due to the increased risk of complications. Tumors adjacent to the gallbladder, in the dome of the liver, and in the caudate lobe have all been described as challenging locations for treatment. Treatment of lesions within $1 \mathrm{~cm}$ of the gallbladder can cause thermal injury to the gallbladder wall leading to cholecystitis. Percutaneous bile aspiration from the gallbladder with or without additional hydrodissection has been shown to allow for safe treatment of lesions in this location. ${ }^{46}$ Infusing nonionic fluid between the diaphragm and the liver, resulting in inferior displacement of the liver, has been shown to aid in the treatment of lesions at the dome. Peritoneal adhesions and tumors located along the bare area limit application of this technique. ${ }^{47}$ Alternatively, intrapleural fluid infusion has been shown to improve ultrasonographic visualization of tumors in the hepatic dome. ${ }^{48}$ Lesions in the caudate lobe are considered difficult to treat for two reasons: first, the long distance from the skin surface to the caudate lobe makes probe placement difficult; second, the caudate is surrounded by several major vessels (inferior vena cava (IVC), portal vein, and hepatic artery). There have been several studies demonstrating the safety of treatment of lesions in this location from an intercostal approach or through the left lobe; however, because of the proximity of the large vessels, a higher rate of local recurrence is reported for these lesions. ${ }^{49}$

\section{Complications}

Percutaneous ablation therapies have low complication rates, especially when compared with surgical interventions. Major and minor complication rates for thermal ablation can be divided into four categories: thermal damage (intestinal perforation, biliary stenosis, grounding pad burns), mechanical damage (hemorrhage, bile leak, pleural effusions, tumor seeding), septic complications (abscess formation, septice- mia, peritonitis), and other (postablation syndrome, cardiac arrest, pulmonary embolism, pneumothorax). ${ }^{50}$

Thermal injuries to the intestinal tract are observed when the treated lesion was within $1 \mathrm{~cm}$ of the liver capsule and adjacent to a gastrointestinal lumen. Prior abdominal surgery or chronic cholecystitis can lead to fibrotic adhesions between the bowel and liver. The biliary tree has also been shown to be susceptible to thermal injury, with treatment of lesions in the hepatic hilum at greatest risk for complication; peripheral biliary strictures are usually not clinically significant. Exophytic lesion location has been associated with higher rates of intraperitoneal hemorrhage. Diabetes and pneumobilia have been shown to be risk factors for septic complications, most commonly presenting as postablation hepatic abscess formation..$^{50}$

Abdominal pain, malaise, fever, nausea, and transient elevation of liver enzymes have been reported with several hepatic tumor ablation techniques and are commonly referred to as postablation syndrome. Symptom onset ranges from 3 to 5 days postprocedure and persists for days to weeks. ${ }^{51}$ The greatest predictor of development of postablation syndrome is the volume of the ablated tumor.

There are a few unique complications reported for cryoablation, namely, myoglobinemia, cryoshock, and "cracking" of the liver. Myoglobinemia tends to be related to the amount of frozen tissue and may cause renal failure. ${ }^{39}$ Cryoshock is a syndrome of multiorgan failure and disseminated intravascular coagulation, and presents in a very similar fashion to septic shock. The etiology is thought to be secondary to necrotic tissue in direct contact with blood vessels immediately after cell death, leading to a systemic reaction. ${ }^{40}$ The concept of liver cracking is a rare but potentially catastrophic hemorrhagic event most often occurring in the thaw phase, and is thought to be due to the air-ice interface as the issue warms.

Mortality rates of $0.4 \%$ have been reported for PEI, up to $0.3 \%$ for RFA, and between 2 and 3.7\% for cryoablation..$^{50,52}$ Major complication rates are also low, ranging from 3.2 to $4.6 \%$ and 2.2 to $3.1 \%$ for PEI and RFA, respectively. The major complication rate reported for cryoablation is slightly higher at $10.8 \%$.

The risk of needle tract seeding remains an ongoing controversy regarding percutaneous ablation, especially in the setting of HCC. Although a low to negligible risk of seeding may be acceptable for patients with unresectable tumors, patients awaiting transplantation could drop off the transplant list if needle tract seeding were to result in peritoneal dissemination of HCC. Early studies in small series reported a $12 \%$ seeding rate after RFA. ${ }^{53}$ Recently, results from a large series of HCC patients treated with RFA demonstrated a much lower incidence of needle tract seeding at $0.5 \%{ }^{50}$ Treatment of tumors in a subcapsular location or with an invasive tumor pattern has been shown to increase the risk of tumor dissemination. ${ }^{50}$

\section{Follow-Up}

Clinical, laboratory, and imaging follow-up is usually performed 1 month after treatment to assess treatment 
response to monitor evolution of the ablated tissue over time, and to evaluate for complications. Periablation hyperemia secondary to an inflammatory reaction and granulation tissue can appear as a concentric, thin rim of enhancement around the ablation zone and is a common finding. With residual tumor, the periablation enhancement appears thick and irregular. Often differentiation of reactive hyperemia from enhancing residual or recurrent tumor is difficult. Close follow-up imaging may be necessary in equivocal cases. ${ }^{54}$

Not surprisingly, new HCC tumors develop in patients on follow-up imaging with the rate of metachronous tumor occurrence, reaching as high as $81 \%$. This is an expression of the inherent multicentric nature of HCC in the setting of cirrhosis $^{11}$; similar recurrence rates can be found in cirrhotic patients with HCC who are treated with surgical resection. ${ }^{55}$

\section{Conclusion}

Image-guided percutaneous ablation is a safe and effective treatment for early HCC and small liver metastases in surgically unresectable patients. Understanding the basic science of the commonly used ablation modalities and knowing the expected clinical outcome will aid in the triage of patients with these malignancies.

\section{References}

1 Clark WL, Morgan D, Asnis EJ. Electrothermic methods in treatment of neoplasms and other lesions with clinical and histological observations. Radiology 1924;2:233-246

2 Livraghi T, Festi D, Monti F, Salmi A, Vettori C. US-guided percutaneous alcohol injection of small hepatic and abdominal tumors. Radiology 1986;161(2):309-312

3 Forner A, Llovet JM, Bruix J. Hepatocellular carcinoma. Lancet 2012;379(9822):1245-1255

4 Alazawi W, Cunningham M, Dearden J, Foster GR. Systematic review: outcome of compensated cirrhosis due to chronic hepatitis C infection. Aliment Pharmacol Ther 2010;32(3):344-355

5 Lencioni R, Crocetti L, Cioni D, Della Pina C, Bartolozzi C. Percutaneous radiofrequency ablation of hepatic colorectal metastases: technique, indications, results, and new promises. Invest Radiol 2004;39(11):689-697

6 Bruix J, Sherman M; Practice Guidelines Committee, American Association for the Study of Liver Diseases. Management of hepatocellular carcinoma. Hepatology 2005;42(5):1208-1236

7 Bruix J, Sherman M; American Association for the Study of Liver Diseases. Management of hepatocellular carcinoma: an update. Hepatology 2011;53(3):1020-1022

8 de Lope CR, Tremosini S, Forner A, Reig M, Bruix J. Management of HCC. J Hepatol 2012;56(Suppl 1):S75-S87

9 Mazzaferro V, Regalia E, Doci R, et al. Liver transplantation for the treatment of small hepatocellular carcinomas in patients with cirrhosis. N Engl J Med 1996;334(11):693-699

10 Wiesner R, Edwards E, Freeman R, et al; United Network for Organ Sharing Liver Disease Severity Score Committee. Model for endstage liver disease (MELD) and allocation of donor livers. Gastroenterology 2003;124(1):91-96

11 Lencioni R, Cioni D, Crocetti L, et al. Early-stage hepatocellular carcinoma in patients with cirrhosis: long-term results of percutaneous image-guided radiofrequency ablation. Radiology 2005; 234(3):961-967
12 Crocetti L, de Baere T, Lencioni R. Quality improvement guidelines for radiofrequency ablation of liver tumours. Cardiovasc Intervent Radiol 2010;33(1):11-17

13 Ahmed M, Brace CL, Lee FT Jr, Goldberg SN. Principles of and advances in percutaneous ablation. Radiology 2011;258(2): 351-369

14 Livraghi T, Giorgio A, Marin G, et al. Hepatocellular carcinoma and cirrhosis in 746 patients: long-term results of percutaneous ethanol injection. Radiology 1995;197(1):101-108

15 Shiina S, Tagawa K, Unuma T, et al. Percutaneous ethanol injection therapy for hepatocellular carcinoma. A histopathologic study. Cancer 1991;68(7):1524-1530

16 Knab LM, Salem R, Mahvi DM. Minimally invasive therapies for hepatic malignancy. Curr Probl Surg 2013;50(4):146-179

17 Lin SM, Lin CJ, Lin CC, Hsu CW, Chen YC. Randomised controlled trial comparing percutaneous radiofrequency thermal ablation, percutaneous ethanol injection, and percutaneous acetic acid injection to treat hepatocellular carcinoma of $3 \mathrm{~cm}$ or less. Gut 2005;54(8):1151-1156

18 Livraghi T, Vettori C, Lazzaroni S. Liver metastases: results of percutaneous ethanol injection in 14 patients. Radiology 1991; 179(3):709-712

19 Lencioni R, Bartolozzi C, Caramella D, et al. Treatment of small hepatocellular carcinoma with percutaneous ethanol injection. Analysis of prognostic factors in 105 Western patients. Cancer 1995;76(10):1737-1746

20 Livraghi T, Bolondi L, Buscarini L, et al; Italian Cooperative HCC Study Group. No treatment, resection and ethanol injection in hepatocellular carcinoma: a retrospective analysis of survival in 391 patients with cirrhosis. J Hepatol 1995;22(5):522-526

21 Gage AA, Baust J. Mechanisms of tissue injury in cryosurgery. Cryobiology 1998;37(3):171-186

22 Goldberg SN, Gazelle GS, Mueller PR. Thermal ablation therapy for focal malignancy: a unified approach to underlying principles, techniques, and diagnostic imaging guidance. AJR Am J Roentgenol 2000;174(2):323-331

23 Livraghi T, Goldberg SN, Lazzaroni S, et al. Hepatocellular carcinoma: radio-frequency ablation of medium and large lesions. Radiology 2000;214(3):761-768

24 Goldberg SN, Hahn PF, Tanabe KK, et al. Percutaneous radiofrequency tissue ablation: does perfusion-mediated tissue cooling limit coagulation necrosis? J Vasc Interv Radiol 1998;9(1 Pt 1): 101-111

25 Choi D, Lim HK, Rhim H, et al. Percutaneous radiofrequency ablation for early-stage hepatocellular carcinoma as a first-line treatment: long-term results and prognostic factors in a large single-institution series. Eur Radiol 2007;17(3):684-692

26 Mahnken AH, Pereira PL, de Baère T. Interventional oncologic approaches to liver metastases. Radiology 2013;266(2):407-430

27 Stang A, Fischbach R, Teichmann W, Bokemeyer C, Braumann D. A systematic review on the clinical benefit and role of radiofrequency ablation as treatment of colorectal liver metastases. Eur J Cancer 2009;45(10):1748-1756

28 Tzeng CW, Aloia TA. Colorectal liver metastases. J Gastrointest Surg 2013;17(1):195-201, quiz 201-202

29 Hur H, Ko YT, Min BS, et al. Comparative study of resection and radiofrequency ablation in the treatment of solitary colorectal liver metastases. Am J Surg 2009;197(6):728-736

30 Lu DS, Raman SS, Limanond P, et al. Influence of large peritumoral vessels on outcome of radiofrequency ablation of liver tumors. J Vasc Interv Radiol 2003;14(10):1267-1274

31 Illing R, Gillams A. Radiofrequency ablation in the treatment of breast cancer liver metastases. Clin Oncol (R Coll Radiol) 2010; 22(9):781-784

32 Bergenfeldt M, Jensen BV, Skjoldbye B, Nielsen D. Liver resection and local ablation of breast cancer liver metastases-a systematic review. Eur J Surg Oncol 2011;37(7):549-557 
33 Adam R, Aloia $\mathrm{T}$, Krissat $\mathrm{J}$, et al. Is liver resection justified for patients with hepatic metastases from breast cancer? Ann Surg 2006;244(6):897-907, discussion 907-908

34 Brace CL. Radiofrequency and microwave ablation of the liver, lung, kidney, and bone: what are the differences? Curr Probl Diagn Radiol 2009;38(3):135-143

35 Lubner MG, Brace CL, Hinshaw JL, Lee FT Jr. Microwave tumor ablation: mechanism of action, clinical results, and devices. J Vasc Interv Radiol 2010;21(8, Suppl):S192-S203

36 Liang P, Dong B, Yu X, et al. Prognostic factors for survival in patients with hepatocellular carcinoma after percutaneous microwave ablation. Radiology 2005;235(1):299-307

37 Dong B, Liang P, Yu X, et al. Percutaneous sonographically guided microwave coagulation therapy for hepatocellular carcinoma: results in 234 patients. AJR Am J Roentgenol 2003;180(6): 1547-1555

38 Shibata T, Iimuro Y, Yamamoto Y, et al. Small hepatocellular carcinoma: comparison of radio-frequency ablation and percutaneous microwave coagulation therapy. Radiology 2002;223(2): 331-337

39 Mala T. Cryoablation of liver tumours-a review of mechanisms, techniques and clinical outcome. Minim Invasive Ther Allied Technol 2006;15(1):9-17

40 Hinshaw JL, Lee FT Jr. Cryoablation for liver cancer. Tech Vasc Interv Radiol 2007;10(1):47-57

41 Littrup PJ, Ahmed A, Aoun HD, et al. CT-guided percutaneous cryotherapy of renal masses. J Vasc Interv Radiol 2007;18(3): 383-392

42 Shimizu T, Sakuhara Y, Abo D, et al. Outcome of MR-guided percutaneous cryoablation for hepatocellular carcinoma. J Hepatobiliary Pancreat Surg 2009;16(6):816-823

43 Adam R, Hagopian EJ, Linhares M, et al. A comparison of percutaneous cryosurgery and percutaneous radiofrequency for unresectable hepatic malignancies. Arch Surg 2002;137(12):1332-1339, discussion 1340

44 Zhang YJ, Liang HH, Chen MS, et al. Hepatocellular carcinoma treated with radiofrequency ablation with or without ethanol injection: a prospective randomized trial. Radiology 2007; 244(2):599-607

45 Ni JY, Liu SS, Xu LF, Sun HL, Chen YT. Meta-analysis of radiofrequency ablation in combination with transarterial chemoembolization for hepatocellular carcinoma. World J Gastroenterol 2013;19(24):3872-3882

46 Levit E, Bruners P, Günther RW, Mahnken AH. Bile aspiration and hydrodissection to prevent complications in hepatic RFA close to the gallbladder. Acta Radiol 2012;53(9):1045-1048

47 Rhim H, Lim HK. Radiofrequency ablation for hepatocellular carcinoma abutting the diaphragm: the value of artificial ascites. Abdom Imaging 2009;34(3):371-380

48 Kondo Y, Yoshida H, Tateishi R, Shiina S, Kawabe T, Omata M. Percutaneous radiofrequency ablation of liver cancer in the hepatic dome using the intrapleural fluid infusion technique. $\mathrm{Br} J$ Surg 2008;95(8):996-1004

49 Nishigaki Y, Tomita E, Hayashi H, et al. Efficacy and safety of radiofrequency ablation for hepatocellular carcinoma in the caudate lobe of the liver. Hepatol Res 2013;43(5):467-474

50 Livraghi T, Solbiati L, Meloni MF, Gazelle GS, Halpern EF, Goldberg SN. Treatment of focal liver tumors with percutaneous radiofrequency ablation: complications encountered in a multicenter study. Radiology 2003;226(2):441-451

51 Dodd GD III, Napier D, Schoolfield JD, Hubbard L. Percutaneous radiofrequency ablation of hepatic tumors: postablation syndrome. AJR Am J Roentgenol 2005;185(1):51-57

52 Kerkar S, Carlin AM, Sohn RL, et al. Long-term follow up and prognostic factors for cryotherapy of malignant liver tumors. Surgery 2004;136(4):770-779

53 Jaskolka JD, Asch MR, Kachura JR, et al. Needle tract seeding after radiofrequency ablation of hepatic tumors. J Vasc Interv Radiol 2005;16(4):485-491

54 Sainani NI, Gervais DA, Mueller PR, Arellano RS. Imaging after percutaneous radiofrequency ablation of hepatic tumors: Part 1 , Normal findings. AJR Am J Roentgenol 2013;200(1):184-193

55 Belghiti J, Panis Y, Farges O, Benhamou JP, Fekete F. Intrahepatic recurrence after resection of hepatocellular carcinoma complicating cirrhosis. Ann Surg 1991;214(2):114-117 